\title{
Prolonged donor heart preservation with pinacidil: The role of mitochondria and the mitochondrial adenosine triphosphate- sensitive potassium channel
}

\author{
Liuqing Yang, MD, a,b and Tian $\mathrm{Yu}, \mathrm{MD}, \mathrm{PhD}^{\mathrm{a}}$
}

\begin{abstract}
Objective: Prolonged donor heart preservation is important in cardiovascular surgery. This study examined the effect of pinacidil, a nonselective adenosine triphosphate-sensitive potassium channel opener, on donor heart preservation when added to histidine tryptophan ketoglutarate solution and the role of mitochondria in this protection.
\end{abstract}

\begin{abstract}
Methods: Sprague-Dawley rat hearts received one of 5 preservation solutions in the Langendorff perfusion apparatus (24 per group): (1) histidine tryptophan ketoglutarate solution; (2) histidine tryptophan ketoglutarate solution containing pinacidil; (3) histidine tryptophan ketoglutarate solution containing pinacidil and 5-hydroxydecanote, a mitochondrial adenosine triphosphate-sensitive potassium channel blocker; (4) histidine tryptophan ketoglutarate solution containing pinacidil and Hoechst-Marion-Roussel 1098, a sarcolemmal adenosine triphosphate-sensitive potassium channel blocker; and (5) histidine tryptophan ketoglutarate solution containing pinacidil, 5-hydroxydecanote, and Hoechst-Marion-Roussel 1098. After a 10-minute equilibration period, all the hearts in the different preservation solutions were placed in cold storage for 8 hours, followed by 60 minutes of reperfusion. Hemodynamics, mitochondrial respiratory function, adenosine triphosphate level, cardiac troponin I release, and ultrastructure were examined.
\end{abstract}

Results: Histidine tryptophan ketoglutarate solution containing $0.5 \mathrm{mmol} / \mathrm{L}$ pinicidal significantly improved heart function, coronary flow, myocardial ultrastructure, and cardiac troponin I release after reperfusion $(P<$ .01 or $P<.05)$. In the pinacidil group at the end of storage and the end of reperfusion, mitochondrial respiratory function and myocardial adenosine triphosphate levels were superior when compared with other groups $(P<.01$ or $P<.05)$. These beneficial effects of pinacidil were blocked by $100 \mu \mathrm{mol} / \mathrm{L}$ 5-hydroxydecanote.

Conclusion: Histidine tryptophan ketoglutarate solution containing pinacidil provides better cardioprotection with preservation of mitochondrial energy. This effect of pinacidil appears to depend on both mitochondrial and sarcolemmal adenosine triphosphate sensitive potassium channel. (J Thorac Cardiovasc Surg 2010;139:1057-63)

Heart transplantation is a common procedure for patients with severe heart failure, and anesthetic management of a donor heart is essential to maintain cardiac function until successful organ transplantation. ${ }^{1}$ Prolongation of global ischemia can be achieved with cardioplegic agents. ${ }^{2}$ Currently, hearts can be stored for a maximum of 4 hours clinically for orthotropic heart transplantation. Patient survival has been inversely correlated with donor heart extracorporeal cold ischemic time. ${ }^{3}$ Histidine tryptophan ketoglutarate (HTK) solution is considered to be superior to the conven-

\footnotetext{
From the Department of Anesthesiology, ${ }^{\mathrm{a}}$ Zunyi Medical College, Zunyi, China, and the Subei People's Hospital of Jiangsu Province, ${ }^{\mathrm{b}}$ Yangzhou, China.

Supported by the Chinese National Natural Science Foundation (no. 30460132).

Disclosures: None.

Received for publication April 2, 2009; revisions received Sept 28, 2009; accepted for publication Oct 25, 2009.

Address for reprints: Tian Yu, MD, PhD, Department of Anesthesiology, Zunyi Medical College, Dalian road 201, Zunyi City, 563000, Guizhou Province, People's Republic of China (E-mail: zyyutian@21cn.com).

$0022-5223 / \$ 36.00$

Copyright (c) 2010 by The American Association for Thoracic Surgery

doi:10.1016/j.jtcvs.2009.10.042
}

tional University of Wisconsin solution in heart preservation and is the ideal solution for organ preservation. ${ }^{4}$

Adenosine triphosphate (ATP)-sensitive potassium (KATP) channels are inwardly rectifying potassium channels regulated by the intracellular level of ATP. Two separate KATP channels are abundantly present in the cardiomyocyte: one is the sarcolemmal KATP (sarcKATP) channel, and the other is the mitochondrial KATP (mitoKATP) channel. ${ }^{5}$ Both KATP channels can play important roles in cardiac protection. ${ }^{6}$ Initial evidence suggests that the sarcKATP channel triggers or mediates the cardioprotective effects of ischemic precondition ${ }^{7-9}$; however, more recent findings have suggested a major role for the mitoKATP channel. The sarcKATP channel can be blocked by Hoechst-Marion-Roussel 1098 (HMR1098), and the mitoKATP channel can be blocked by 5-hydroxydecanote (5HD). Pinacidil is a nonselective KATP channel opener, which has been shown to have significant cardioprotective effects. ${ }^{10,11}$ Pinacidil can open both the sarcolemmal and mitochondrial KATP channels, leading to cell membrane hyperpolarization and mitochondrial membrane depolarization. Hyperkalemic arrest has been shown to be associated 


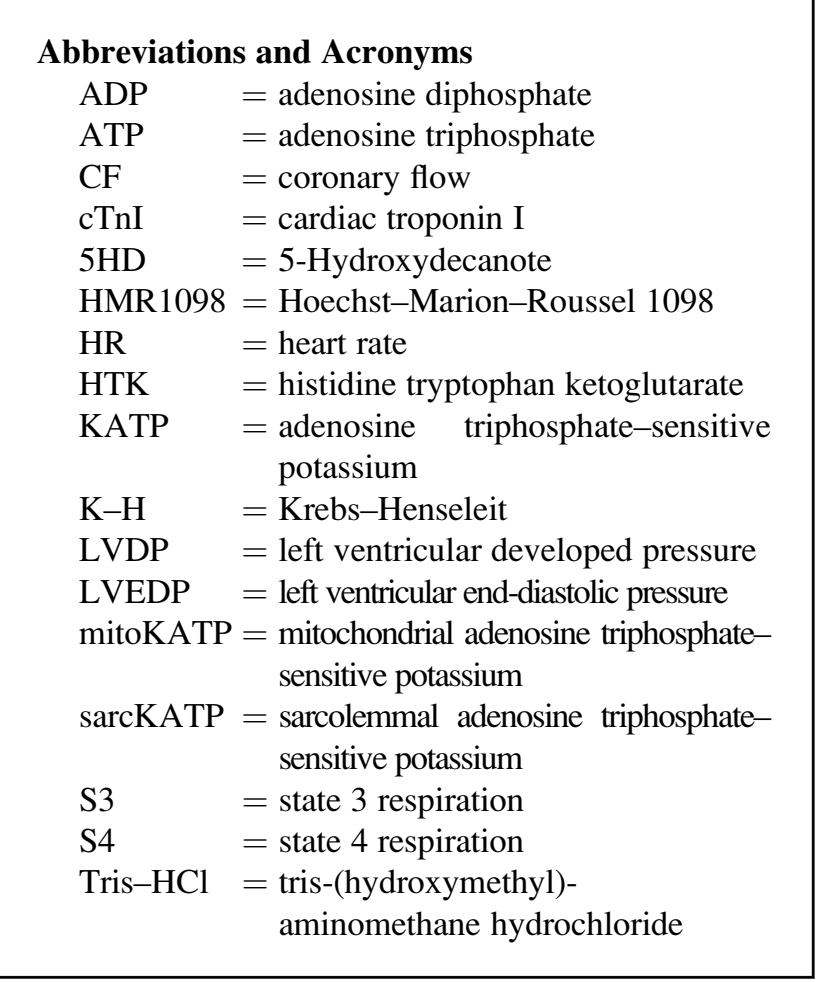

with decreased myocardial ATP levels compared with those seen after hyperpolarization arrest with pinacidil. ${ }^{12}$

Based on the literature, we hypothesize that the combination of HTK solution with pinacidil would be a better strategy for donor heart preservation. The current study was therefore designed to test this hypothesis by using isolated rat hearts. To examine the role of KATP channel openers in cardiac protection, HMR 1098 and 5HD were included in separate groups to block the effects of pinacidil. Complete heart functions are compared after prolonged storage, and mitochondrial respiratory function and ATP production are measured. Furthermore, transmission electron microscopy is used to examine the ultrastructural changes under different donor heart preservation conditions.

\section{MATERIALS AND METHODS \\ Chemicals and Equipment}

Pinacidil, 5HD, ATP, adenosine diphosphate (ADP), sodium succinate, tris-(hydroxymethyl)-aminomethane hydrochloride (Tris-HCl), hydroxyethylpiperazine ethanesulfonic acid, and HTK solution were purchased from Sigma-Aldrich Corporation (St Louis, Mo); HMR 1098 was a generous gift from Aventis Pharmaceuticals (Frankfurt, Germany); the troponin I kit was purchased from DPC Corporation (Los Angeles, Calif); all others were analytically pure made in China. The Langendorff perfusion models and Powerlab physioexperimental system were purchased from AD Instruments (Bella Vista, New South Wales, Australia); the TECNAI10 electron microscope was purchased from Phillips (Eindhoven, The Netherlands); the Shimadzu-10AVP high-performance liquid chromatographic apparatus was purchased from Shimadzu (Tokyo, Japan); the biologic apparatus for determining oxygen was purchased from Hengte (Beijing, China); and the Immu- lite 1000 chemiluminescence immune essay apparatus was purchased from DPC Corporation.

\begin{abstract}
Animals
Sprague-Dawley rats of either sex, weighing 250 to $350 \mathrm{~g}$, were provided by the Center of Laboratory Animals in Third Military Medical University (Chongqing, China). All animals received humane care in compliance with the "Guide for the care and use of laboratory animals" in China (no. 14924, 2001) and allowed free access to rodent chow and tap water. All animal surgical procedures were approved by the institutional animal care and use committee.
\end{abstract}

\section{Isolated Heart Perfusion Model}

Anesthesia was accomplished through an intraperitoneal injection of sodium pentobarbital (Sagatal; $65 \mathrm{mg} / \mathrm{kg}$ ) and heparin (1.4 U/g). The rat hearts after sternotomy and rapid cardiectomy were put in precooling Krebs-Henseleit $(\mathrm{K}-\mathrm{H})$ buffer $\left(4^{\circ} \mathrm{C}\right)$, and the aorta was cannulated and rapidly attached to a perfusion column in $\mathrm{K}-\mathrm{H}$ solution $\left(37^{\circ} \mathrm{C}\right)$ buffered with a gas mixture of $95 \% \mathrm{O}_{2}$ and $5 \% \mathrm{CO}_{2}$ for 60 seconds at a constant pressure of $7.5 \mathrm{~mm} \mathrm{Hg}$ as regression perfusion. After beating heart recovery, a microstomia cut was made at the end of the pulmonary artery and left atrial appendage, and a fluid-filled latex balloon connected to a fluid-filled transducer was inserted into the left ventricle through the mitral valve and attached to the Powerlab system. Left ventricular end-diastolic pressure (LVEDP) was controlled between 7 and $9 \mathrm{~mm} \mathrm{Hg}$ through the fluid-filled latex balloon, and the balloon volume was not adjusted after the baseline value was obtained. Heart rate (HR), LVEDP, left ventricular developed pressure (LVDP), and coronary flow $(\mathrm{CF})$ were recorded.

\section{Experimental Design}

After a 10 -minute equilibration period in $\mathrm{K}-\mathrm{H}$ solution $\left(37^{\circ} \mathrm{C}\right)$, the isolated hearts were randomly allocated into 5 groups $(n=24$ per group). Each group was then divided into 3 subgroups at different time points: the end of equilibration, the end of storage, and the end of reperfusion. Hearts from group 1 were arrested in HTK solution. Hearts from Group 2 were arrested in HTK solution containing pinacidil $(0.5 \mathrm{mmol} / \mathrm{L})$. Hearts from group 3 were arrested in HTK solution containing pinacidil and 5HD, a selective mitochondrial KATP channel blocker $(100 \mu \mathrm{mol} / \mathrm{L})$. Hearts from group 4 were arrested in HTK solution containing pinacidil and HMR 1098, a selective sarcolemmal KATP channel blocker (HMR1098, $100 \mu \mathrm{mol} / \mathrm{L}$ ). Finally, hearts from group 5 were arrested in HTK solution containing pinacidil, 5HD, and HMR1098. The dose of arresting solution was $20 \mathrm{~mL} / \mathrm{kg}$ at $10 \mathrm{~mL} / \mathrm{min}$ and $4^{\circ} \mathrm{C}$. After all arrested hearts were placed into cold storage at $4^{\circ} \mathrm{C}$ for 8 hours, a 60 -minute reperfusion was performed in oxygenated $\mathrm{K}-\mathrm{H}$ solution at $37^{\circ} \mathrm{C}$. Heart function parameters at the end of equilibration and reperfusion were recorded, and coronary effluents were collected at the end of equilibration and 20 minutes after reperfusion and stored at $-80^{\circ} \mathrm{C}$ before analysis. Approximately $100 \mathrm{mg}$ of myocardium was removed and quick frozen in liquid nitrogen for later ATP measurement.

\section{Extraction of Myocardial Mitochondria}

Myocardial mitochondria were isolated at the end of the equilibration, storage, and reperfusion. Briefly, the isolated hearts were rapidly cut into pieces in $4^{\circ} \mathrm{C}$ precooling mitochondrion-separating medium containing $225 \mathrm{mmol} / \mathrm{L}$ mannitol, $75 \mathrm{mmol} / \mathrm{L}$ sucrose, $10 \mathrm{mmol} / \mathrm{L}$ Tris- $\mathrm{HCl}$, and 1 $\mathrm{mmol} / \mathrm{L}$ ethylenediamine tetraacetic acid at $\mathrm{pH} 7.4$ and were dissociated by the high-speed disperser, followed by homogenization with the precooling glass homogenizer. A $10 \%(\mathrm{wt} / \mathrm{vol})$ myocardial homogenate was made and centrifuged at $600 \mathrm{~g}$ for 7 minutes, the supernatant of which was centrifuged again at $10,000 \mathrm{~g}$ for 11 minutes. The precipitated mitochondria were then washed with the separating medium and finally suspended by the 
dispersing medium, with $150 \mathrm{mmol} / \mathrm{L} \mathrm{KCl}, 5 \mathrm{mmol} / \mathrm{L}$ Tris- $\mathrm{HCl}$, and 1 $\mathrm{mmol} / \mathrm{L}$ ethylenediamine tetraacetic acid at $\mathrm{pH}$ 7.4. The isolation procedure was kept at approximately $0^{\circ} \mathrm{C}$ to $4^{\circ} \mathrm{C}$ and finished in an hour. The Bradford method was used for mitochondrial protein quantization, and the protein levels were kept in the range of 1.0 to $2.0 \mathrm{mg} / \mathrm{mL}$.

\section{Determination of Mitochondrial Respiratory Function}

Mitochondrial respiratory function was determined by using Clark-mode electrodes in a 3-mL temperature-controlled oxygen measurement pool according to the literature. ${ }^{12,13}$ The volume of reaction solution was $2.5 \mathrm{~mL}$ at a temperature of $30^{\circ} \mathrm{C}$. Briefly, $1.9 \mathrm{~mL}$ of determination solution containing $220 \mathrm{mmol} / \mathrm{L}$ D-mannitol, $70 \mathrm{mmol} / \mathrm{L}$ sucrose, $2 \mathrm{mmol} / \mathrm{L}$ hydroxyethylpiperazine ethanesulfonic acid, and $\mathrm{KH}_{2} \mathrm{PO}_{4}$ at $\mathrm{pH} 7.0$ was added. After an equilibration period of approximately 15 to 20 minutes, $0.5 \mathrm{~mL}$ of mitochondrial suspension was added, and approximately 20 to 30 minutes of the oxygen consumption curve were recorded. Fifty microliters of $250 \mathrm{mmol} / \mathrm{L}$ amber acid was not added until the curve was stable; hence state 4 respiration (S4) was started. In 1 or 2 minutes, $50 \mu \mathrm{L}$ of $100 \mathrm{mmol} / \mathrm{L}$ ADP was added to start state 3 respiration (S3). The variation of oxygen partial pressure was recorded; the S3 and S4 oxygen consumption per unit time per unit of mitochondrial protein was calculated (in nanomoles per minutes per milligram of protein).

\section{Determination of Myocardial ATP Levels}

The myocardial tissue was taken out of liquid nitrogen, weighed exactly, and then put into the precooling grinder, where $0.4 \mathrm{~mol} / \mathrm{L} \mathrm{HClO}_{4}$ was added to make $5 \%$ homogenates, followed by centrifugation at $10,000 \mathrm{~g}$ for 20 minutes. The supernatant was centrifuged again at $10,000 \mathrm{~g}$ for 20 minutes in the $\mathrm{pH}$ range of 6.0 to 7.0 , adjusted by $1 \mathrm{~mol} / \mathrm{L} \mathrm{K}_{2} \mathrm{HPO}_{4}$. The supernatant was then used for chromatographic analysis. The above procedures were conducted at $4^{\circ} \mathrm{C}$. The chromatographic conditions were as follows: Kromasil C18 chromato bar $(150 \mathrm{~mm} \times 4.6 \mathrm{~mm}$ id $5 \mu \mathrm{m}$ [Akzonobel, Amsterdam, The Netherlands]); column temperature, $25^{\circ} \mathrm{C}$; mobile phase, buffered phosphate at $\mathrm{pH} 7.0$; absorbance units full scale, 0.05 absorbance units; flow rate, $1 \mathrm{~mL} / \mathrm{min}$; detection wavelength, $254 \mathrm{~nm}$; and sample size, 10 $\mu \mathrm{L}$. The ATP chromatographic peak of the detection sample was determined in reference to the ATP standards. The level of ATP was determined based on the ratio of the peak area of standard to sample.

\section{Determination of Cardiac Troponin I Release}

Cardiac troponin I (cTnI) release in coronary effluent was determined by using the Immulite 1000 chemiluminescence immune assay apparatus with commercial cTnI kits from DPC Corporation.

\section{Electron Microscopy}

One cubic millimeter of myocardial structure of the left ventricle at the end of equilibration and reperfusion in different groups was fixed in $3 \%$ paraformaldehyde and $0.25 \%$ glutaraldehyde solution, mounted, and flushed, followed by $1 \%$ osmic acid for mounting, acetone for dehydration, and ethoxyline 618 for embedment. Extrathin sections were made and photographed with an electron microscope at $10,000 \times$ magnification. The extent of ultrastructural damage was quantified by using Flameng's score. ${ }^{14}$ For each sample, 5-field views were randomly selected, and 20 mitochondria were selected per 5-field view. The criteria for mitochondrial damage were as follows: grade 0 , the granules of mitochondria were integrated, with normal structure; grade 1 , the granules of mitochondria were lost, with normal structure; grade 2, the granules of mitochondria were lost, with swelling mitochondria and limpid matrix; grade 3, the granules of mitochondria were lost, with limpid matrix and ruptured cristae; and grade 4, the granules of mitochondria were lost, with ruptured cristae but short of complete inner and external membranes.

\section{Statistical Analysis}

Statistical analysis was performed with SPSS 12.0 software (SPSS, Inc, Chicago, Ill). Results were expressed as means \pm standard deviations. Oneway analysis of variance was used to compare measurement data among groups, and a paired $t$ test was used to compare the data obtained before and after prolonged donor heart storage.

\section{RESULTS \\ Heart Function}

There were no significant differences in HR, LVEDP, LVDP, and CF at the end of equilibration among the groups. Pinacidil in HTK solution showed more beneficial effects in improving heart function than HTK solution alone (Figure 1), with faster HR, higher LVDP and CF, and lower LVEDP. The beneficial effects of pinacidil could be partially blocked by 5 HD and HMR 1098 and were completely abolished when both blockers were applied (Figure 1).

\section{Mitochondrial Respiratory Function}

There was no significant difference in respiratory control ratio, the ratio of ADP to oxygen atom, and $\mathrm{S} 3$ at the end of equilibration among all the groups. At the end of storage or reperfusion, the levels of respiratory control ratio, the ratio of ADP to oxygen atom, and S3 were decreased markedly $(P<.05)$. Mitochondrial respiratory function parameters in the pinacidil group were higher than those in other groups $(P<.05)$, suggesting better protection. The beneficial effects of pinacidil were completely abolished when both blockers were applied (Tables 1 and 2).

\section{Myocardial ATP Levels}

At the end of reperfusion, the myocardial ATP levels in the pinacidil group were much higher than those in the other groups $(P<.05)$. Additionally, the levels in the 5HD and HMR1098 groups after reperfusion were higher than that in the HTK group $(P<.05)$, and when both 5HD and HMR1098 were applied, the beneficial effects of pinacidil were abolished (Figure 2).

\section{cTnI Levels}

At the end of reperfusion, cTnI levels were increased, which is indicative of heart injury. Pinacidil administration reduced cTnI levels $(P<.05)$, which is consistent with cardioprotection. 5HD and HMR1098 partially blocked pinacidil's effects, but the cTnI release was still lower than that seen in the HTK-only group. When both 5HD and HMR1098 were used together, pinacidil's effects were abolished (Figure 3).

\section{Electron Microscopy}

In the HTK-only group damaged cell membrane was evident, myocardial fibers were in disorder, myocomma structure was unclear, and parts of the myofilament were broken or dissolved. Mitochondria were moderately swollen; cristae were disposed in disorder and dimness, with 

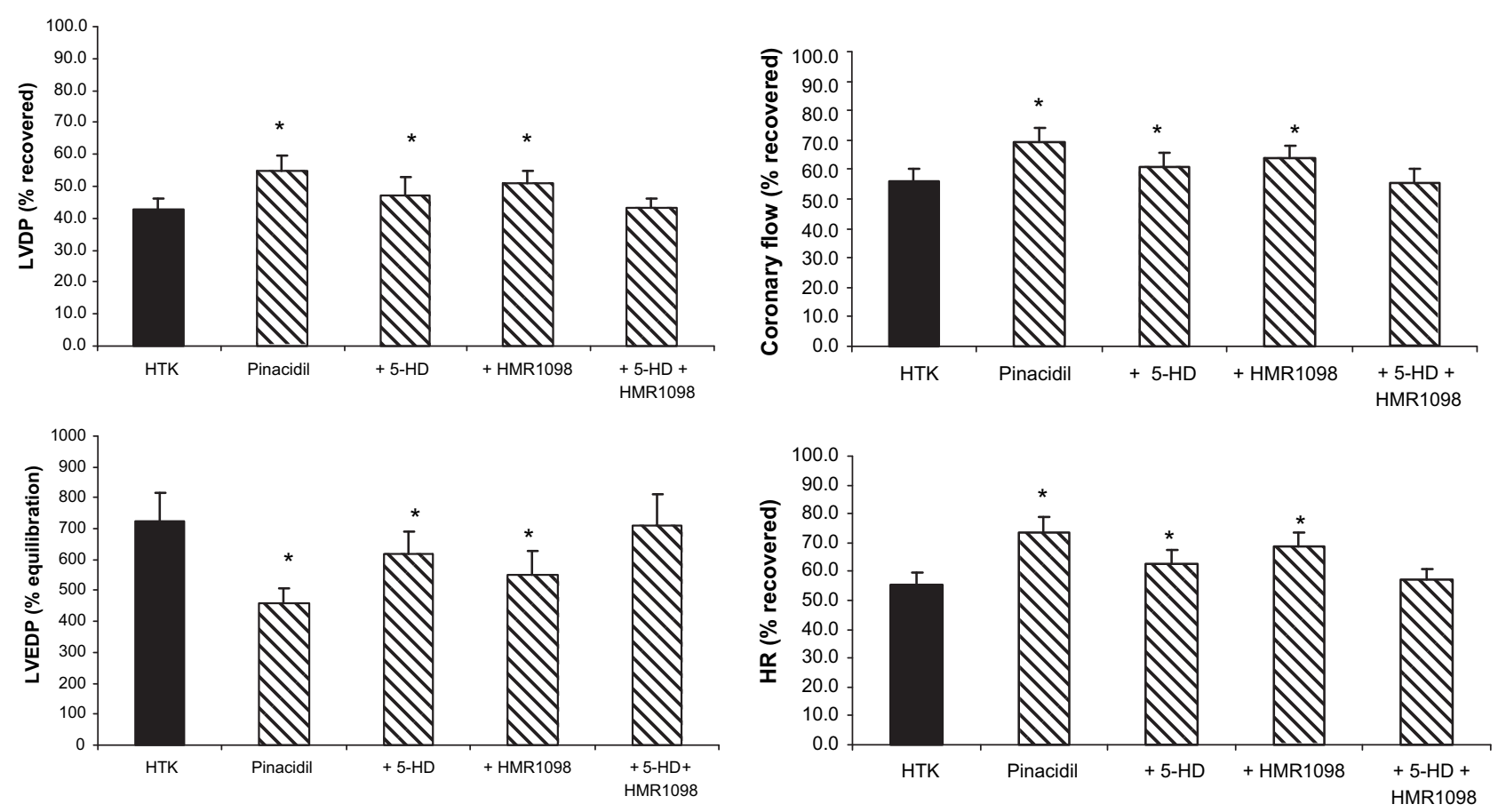

FIGURE 1. Recovery of heart rate $(H R)$, coronary flow $(C F)$, left ventricular developed pressure $(L V D P)$, and left ventricular end-diastolic pressure (LVEDP) 60minutes after reperfusion. Rat hearts were arrested with histidine tryptophan ketoglutarate only (HTK), pinacidil, HTK solution containing pinacidil $(0.5 \mathrm{mmol} / \mathrm{L})$, pinacidil solution plus 5-hydroxydecanote $(5-H D ; 100 \mu \mathrm{mol} / \mathrm{L})$, pinacidil solution plus Hoechst-Marion-Roussel $1098(100 \mu \mathrm{mol} / \mathrm{L})$, and pinacidil solution plus 5-HD and HMR1098. Hearts were subjected to cold storage for 8hours, followed by reperfusion with oxygenated Krebs-Henseleit solution. Data are presented as the mean \pm standard error of the mean. $* P<.05$ versus HTK solution only.

vacuolar degeneration and rupture. In some areas cristae had disappeared, and matrix was lost (Figure 4, A). The HTK solution containing pinacidil showed much better morphology, the cell membrane was intact, myocardial fibers were in order, and myocommal structure was clear. Mitochondria were only slightly swollen, with intact appearance. Nuclei and chromatin were not transformed (Figure 4, B); when the mitochondrial potassium channel blocker 5HD was added, the myocardial damage was intensified (Figure 4, $C$ ). When the selective sarcolemmal KATP channel blocker HMR1098 was added, the myocardial damage was mild compared with that seen with HTK solution alone: the cell membrane appeared to be integrated, myocardial fibers were in order, myocommal structure was clear, and only foci of broken or dissolved myofilaments were evident, with slightly swollen mitochondria (Figure 4, D). When both 5HD and HMR1098 were added, myocardial damage was similar to that seen with HTK solution alone, and the protection provided by pinacidil disappeared (Figure 4, E).

Quantization of mitochondrial ultrastructure changes, as determined by using Flameng's score, is shown in Figure 5. Flameng's score was more than doubled at the end of cardiac reperfusion, from 1.15 to 2.76 , and was decreased by pinacidil to 1.58. 5HD and HMR1098 partially offset pinacidil's effects, bringing scores back to 2.21 and 1.98 , respectively,

TABLE 1. Mitochondrial respiratory control ratio and the ratio of adenosine diphosphate to oxygen atom at the end of equilibration, the end of 8 hours of cold storage, and the end of 60 minutes of reperfusion

\begin{tabular}{|c|c|c|c|c|c|c|}
\hline \multirow[b]{2}{*}{ Group } & \multicolumn{2}{|c|}{ End of equilibration } & \multicolumn{2}{|c|}{ End of 8-h storage } & \multicolumn{2}{|c|}{ End of $60-\mathrm{min}$ reperfusion } \\
\hline & RCR & ADP/O & RCR & ADP/O & RCR & ADP/O \\
\hline HTK & $2.89 \pm 0.05$ & $1.84 \pm 0.03$ & $2.16 \pm 0.07$ & $1.34 \pm 0.08$ & $1.31 \pm 0.04$ & $1.03 \pm 0.07$ \\
\hline Pinacidil & $2.93 \pm 0.05$ & $1.86 \pm 0.05$ & $2.51 \pm 0.04 *$ & $1.54 \pm 0.03^{*}$ & $1.56 \pm 0.06^{*}$ & $1.21 \pm 0.03 *$ \\
\hline$+5 \mathrm{HD}$ & $2.88 \pm 0.04$ & $1.85 \pm 0.03$ & $2.22 \pm 0.05^{*}$ & $1.40 \pm 0.06^{*}$ & $1.39 \pm 0.04 *$ & $1.10 \pm 0.04 *$ \\
\hline+1098 & $2.90 \pm 0.05$ & $1.87 \pm 0.05$ & $2.41 \pm 0.05^{*}$ & $1.46 \pm 0.03^{*}$ & $1.47 \pm 0.06^{*}$ & $1.15 \pm 0.04 *$ \\
\hline$+5 \mathrm{HD}+1098$ & $2.92 \pm 0.04$ & $1.84 \pm 0.04$ & $2.16 \pm 0.05$ & $1.33 \pm 0.04$ & $1.30 \pm 0.04$ & $1.02 \pm 0.08$ \\
\hline
\end{tabular}

Data are presented as the mean \pm standard error of the mean. $R C R$, Respiratory control ratio; $A D P / O$, ADP to oxygen atom ratio; $H T K$, histidine tryptophan ketoglutarate; $5 H D$, 5-hydroxydecanote; 1098, Hoechst-Marion-Roussel $1098 . * P<.05$ versus HTK solution only. 
TABLE 2. Mitochondrial state 4 and state 3 respiration function at the end of equilibration, the end of 8 hours of cold storage, and the end of 60 minutes of reperfusion

\begin{tabular}{|c|c|c|c|c|c|c|}
\hline \multirow[b]{2}{*}{ Group } & \multicolumn{2}{|c|}{ End of equilibration } & \multicolumn{2}{|c|}{ End of 8-h storage } & \multicolumn{2}{|c|}{ End of 60-min reperfusion } \\
\hline & RCR & ADP/O & RCR & ADP/O & RCR & ADP/O \\
\hline HTK & $2.89 \pm 0.05$ & $1.84 \pm 0.03$ & $2.16 \pm 0.07$ & $1.34 \pm 0.08$ & $1.31 \pm 0.04$ & $1.03 \pm 0.07$ \\
\hline Pinacidil & $2.93 \pm 0.05$ & $1.86 \pm 0.05$ & $2.51 \pm 0.04 *$ & $1.54 \pm 0.03 *$ & $1.56 \pm 0.06^{*}$ & $1.21 \pm 0.03^{*}$ \\
\hline$+5 \mathrm{HD}$ & $2.88 \pm 0.04$ & $1.85 \pm 0.03$ & $2.22 \pm 0.05^{*}$ & $1.40 \pm 0.06^{*}$ & $1.39 \pm 0.04^{*}$ & $1.10 \pm 0.04^{*}$ \\
\hline+1098 & $2.90 \pm 0.05$ & $1.87 \pm 0.05$ & $2.41 \pm 0.05^{*}$ & $1.46 \pm 0.03 *$ & $1.47 \pm 0.06^{*}$ & $1.15 \pm 0.04 *$ \\
\hline$+5 \mathrm{HD}+1098$ & $2.92 \pm 0.04$ & $1.84 \pm 0.04$ & $2.16 \pm 0.05$ & $1.33 \pm 0.04$ & $1.30 \pm 0.04$ & $1.02 \pm 0.08$ \\
\hline
\end{tabular}

Data are presented as the mean \pm standard error of the mean. $R C R$, Respiratory control ratio; $A D P / O$, ADP to oxygen atom ratio; $H T K$, histidine tryptophan ketoglutarate; $5 H D$, 5-hydroxydecanote; 1098, Hoechst-Marion-Roussel 1098. *P<.05 versus HTK solution only.

and when both were applied together, they abolished pinacidal's effects, with a score of 2.82 .

\section{DISCUSSION}

The present study demonstrated that HTK solution containing pinacidil provides much better cardioprotection than HTK solution alone, as evidenced by heart function, hemodynamics, mitochondrial respiratory function, ATP levels, and myocardial enzyme levels. The transmission electron microscopic examination of the myocardial ultrastructure further confirmed these results. Hearts stored with HTK solution containing pinacidil can last as long as 8 hours compared with the conventional 4 hours. By using the KATP channel blockers 5HD and HMR1098, the present study demonstrated that both mitoKATP and sarcKATP channels are likely involved in pinacidil's effects, which is consistent with the theory of Diodato and colleagues. ${ }^{11}$

An important ATP-consuming mechanism unique to cardiomyocytes is the actin-myosin interaction. Although the

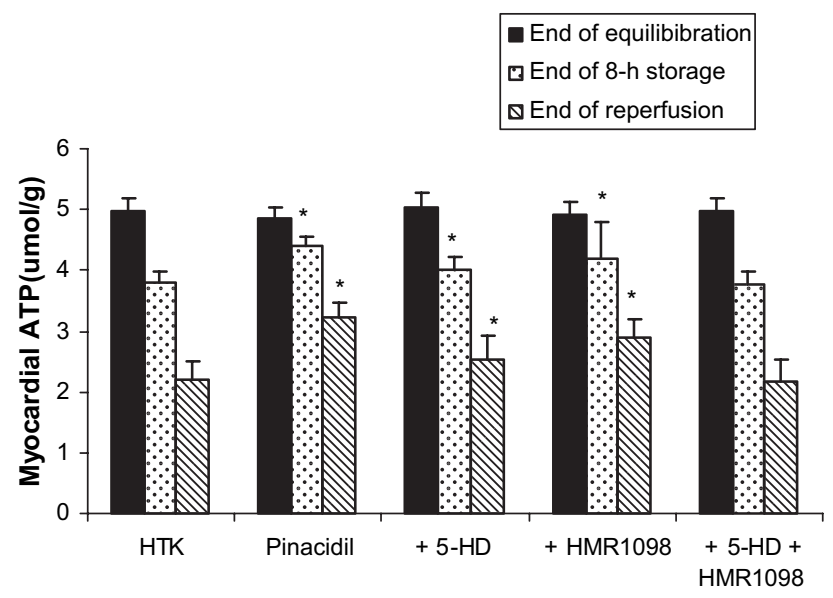

FIGURE 2. Myocardial adenosine triphosphate $(A T P)$ levels 60 minutes after reperfusion. Rat hearts were arrested with histidine tryptophan ketoglutarate $(H T K)$, pinacidil, pinacidil plus 5-hydroxydecanote (5- $H D)$, pinacidil plus Hoechst-Marion-Roussel 1098 (HMR1098), and pinacidil plus 5-HD and HMR1098, as detailed in Figure 1. Hearts were subjected to cold storage for 8 hours, followed by reperfusion. Data are presented as the mean \pm standard error of the mean. $* P<.05$ versus HTK solution only. heart is mechanically arrested, this interaction continues at a low level because the energy for metabolism is very limited in the absence of oxygen. Our previous study demonstrated that pinacidil-containing hyperpolarizing cardioplegia was superior to hyperkalemia-induced depolarized arrest in preserving heart energy and cardiac function in dogs. ${ }^{10}$ Thus preservation of energy-rich phosphate in the myocardial tissue is the key for heart recovery. The present study has proved that donor hearts preserved with HTK containing pinacidil show minimal ATP consumption. This might be due to a better-preserved mitochondrial function and structure, or the sarcKATP channel opened by pinacidil could result in hyperpolarized arrest, with little metabolic demand and inactivation of ion channels. In our previous studies, ${ }^{12}$ isolated rabbit hearts treated with $50 \mu \mathrm{mol} / \mathrm{L}$ pinacidil for hyperpolarized arrest and $10 \mu \mathrm{mol} / \mathrm{L}$ pinacidil for preconditioning showed better energy and improved myocardial metabolism after postischemic reperfusion.

The HTK solution containing pinacidil can also prevent calcium overload. Baczkó and colleagues ${ }^{15}$ perfused the cardiocyte surface with $\mathrm{K}^{+}$ion at different levels, immediately followed by the treatment of hypoxia and recovery, and they

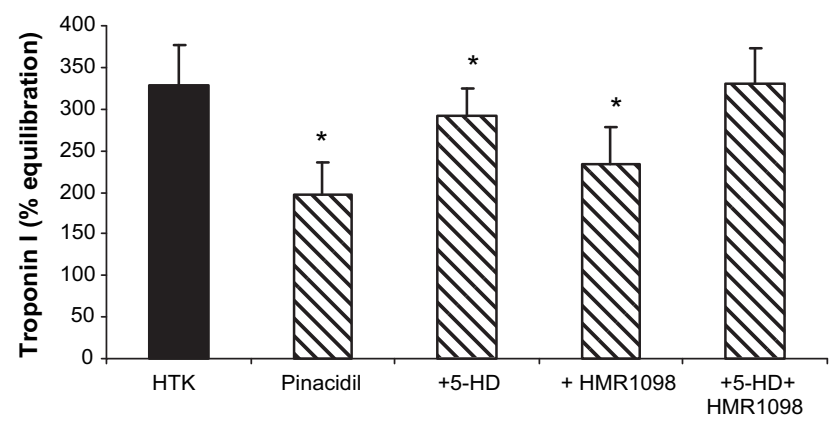

FIGURE 3. Cardiac troponin I levels 60 minutes after reperfusion. Rat hearts were arrested with histidine tryptophan ketoglutarate (HTK), pinacidil, pinacidil plus 5-hydroxydecanote (5-HD), pinacidil plus Hoechst-Marion-Roussel 1098 (HMR1098), and pinacidil plus 5-HD and HMR1098, as detailed in Figure 1. Hearts were subjected to cold storage for 8 hours, followed by reperfusion. Cardiac troponin I levels were expressed as percentage of equilibration. Data are presented as the mean \pm standard error of the mean. $* P<.05$ versus HTK solution only. 

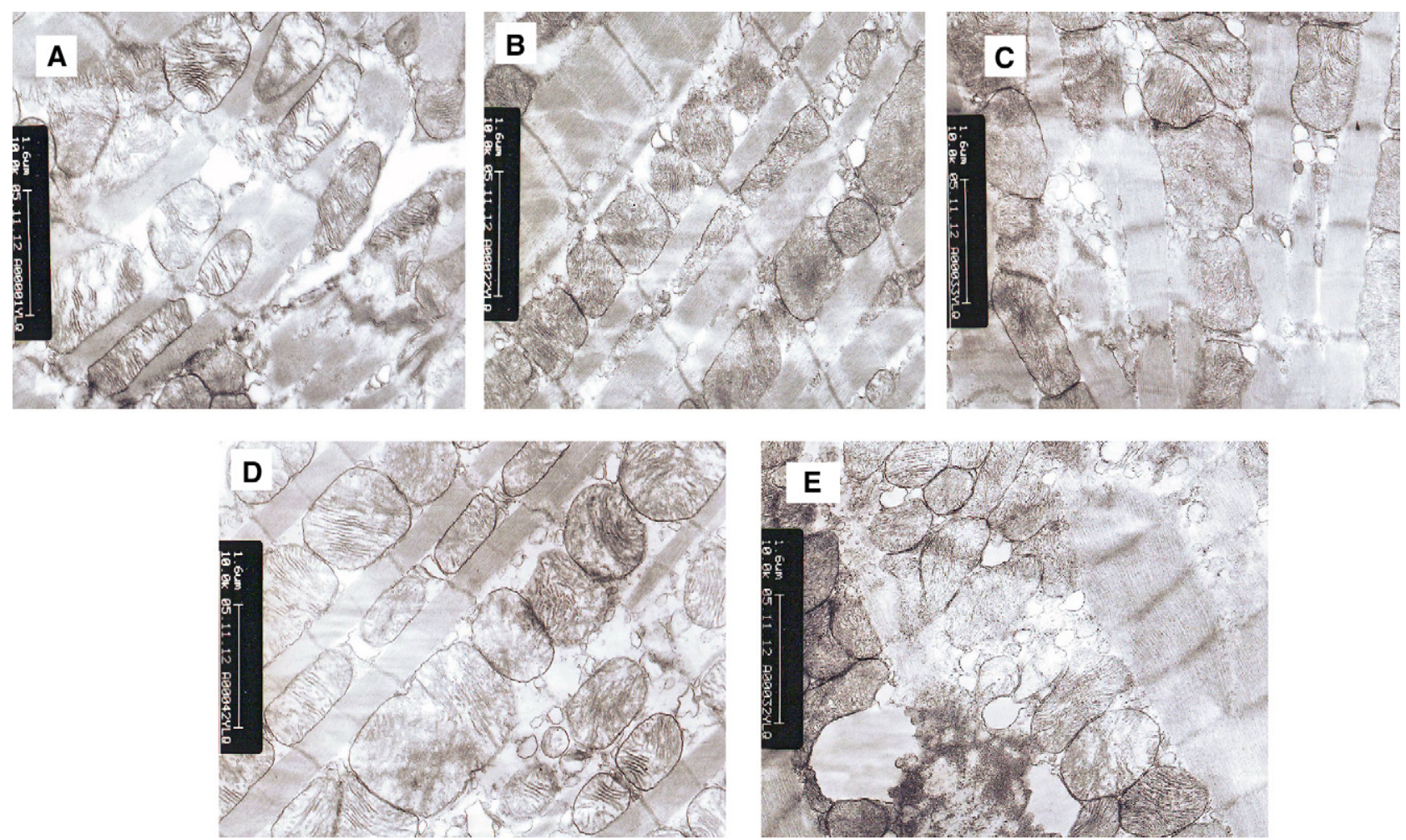

FIGURE 4. Representative myocardial transmission electron microscopic images $(10,000 \times$ magnification $)$ of rat hearts at the end of reperfusion. Rat hearts were arrested with histidine tryptophan ketoglutarate (HTK) solution (A), HTK solution containing pinacidil (B), pinacidil solution plus 5-hydroxydecanote (5-HD; C), pinacidil solution plus Hoechst-Marion-Roussel 1098 (HMR-1098; D), or pinacidil solution plus 5-HD and HMR1098 (E) and subjected to 8 hours of cold storage, followed by 60 minutes of reperfusion.

found that intracellular $\mathrm{Ca}^{2+}$ ion degraded significantly at the state of hyperpolarized membrane potential $\left(\mathrm{K}^{+}, 2.5 \mathrm{mmol} /\right.$ L). It might be due to the fact that the abatement of calcium overload suppresses myocardial contraction with ischemia

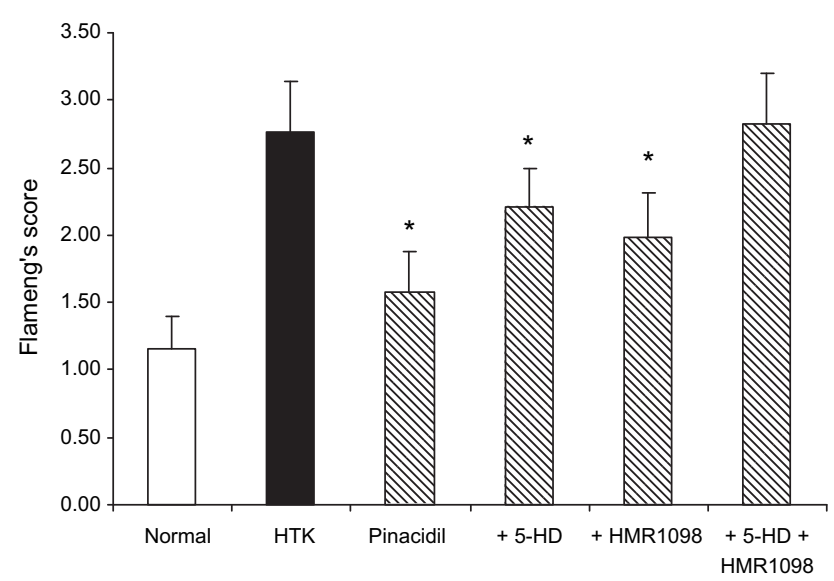

FIGURE 5. Flameng's score of mitochondria observed by means of electron microscopy. Extrathin sections were performed at the end of equilibration and at the end of reperfusion of the histidine tryptophan ketoglutarate (HTK), pinacidil, pinacidil plus 5-hydroxydecanote (5-HD), pinacidil plus Hoechst-Marion-Roussel 1098 (HMR1098), and pinacidil plus 5-HD and HMR 1098 groups. Data are presented as the mean \pm standard error of the mean. $* P<.05$ versus HTK solution or the end of equilibration. and the vascular and myocardial tension diminishes, thus reducing ATP consumption. Furthermore, cardiac and coronary relaxation is advantageous to cardiac recovery. "Mirror changes",16 exist in calcium overload in mitochondria and myocytes, and the opening of the sarcKATP channel can lessen calcium overload and protect mitochondria. Additionally, HTK solution containing pinacidil can relax cardiovascular smooth muscle. ${ }^{17}$ Coronary KATP channel activity might contribute to the regulation of resting myocardial blood flow in patients with congestive heart failure, ${ }^{18}$ and a vasodilator state could result in more energy supply.

In addition, pinacidil can open the mitoKATP channel, leading to mitochondrial membrane depolarization, to provide better ATP preservation. When the mitoKATP channel opens, the transmembrane potential difference decreases, and the inflow motivation of the $\mathrm{Ca}^{2+}$ ion diminishes. Mitochondrial calcium overload usually results in swelling of mitochondria, opening of the permeability transition pore, and deprivation of the initial electrochemical gradient $(\Delta \psi){ }^{19}$ Inflow of $\mathrm{K}^{+}$ion could result in an increase in mitochondrial osmotic pressure and swelling of the mitochondrial matrix. It is reported that an appropriate increase in mitochondrial volume (within $10 \%$ ) can activate fatty acid oxidation and electron transfer, and increase of the junction of the ental and ectal membranes is profit for ADP transfer to promote 
ATP production. ${ }^{20}$ Furthermore, diazoxide, a specific mitoKATP channel opener, can help maintain mitochondrial integrity and suppresses apoptosis. ${ }^{21}$ The mitoKATP channel also plays a critical role in the myocardial protection induced by remote ischemic preconditioning. ${ }^{22}$ The opening of the mitochondrial permeability transition pore and the release of cytochrome $\mathrm{C}$ are 2 key events during apoptosis. ${ }^{23}$ Thus the intervention targeting mitochondria and the mitoKATP channel is promising. ${ }^{7}$

The sarcKATP channel is a sensor for myocardial energy status, ${ }^{8}$ and its opening is determined by intracellular ATP concentrations. Hypoxia, activation of sarcKATP channels in sinoatrial node cells, inhibits sinoatrial node automaticity and is important for the protection of sinoatrial node cells. ${ }^{9}$ The sarcKATP channel triggers delayed ischemic preconditioning in rats. ${ }^{24}$ Some researchers ${ }^{25}$ have observed that removal of the sarcKATP channel-expressed gene made mice insensitive to ischemic preconditioning, whereas the protection of ischemic preconditioning is correlated mainly with activation of the mitoKATP channel, demonstrating that there might be complementary or synergistic action existing between the sarcKATP and mitoKATP channels. Further study is needed to elucidate the effects and correlations between the sarcKATP and mitoKATP channels.

Myocyte structure is the basis of heart function. In this study the electron microscopic pictures display that the myocardial and mitochondrial integrity of the heart stored with HTK solution containing pinacidil are superior to those of HTK solution alone. Cardiomyocyte damage from the heart stored with HTK containing pinacidil was also diminished, as evidenced by serum cardiac-specific cTnI levels.

In conclusion, HTK solution containing pinacidil can improve cardioprotection with better energy preservation, and both the sarKATP and mitoKATP channels play important roles in myocardial protection. An ideal preservation solution should provide prolonged, safe, and predictable preservation of donor organs. The addition of pinacidil to HTK solution can improve myocardial recovery after deep hypothermia and prolong ischemic storage and could have applications in prolonged heart donor preservation in the future.

\section{References}

1. Hunt SA, Haddad F. The changing face of heart transplantation. J Am Coll Cardiol. 2008;52:587-98.

2. Hoenicke EM, Sun X, Strange RG Jr, Damiano RJ Jr. Donor heart preservation with a novel hyperpolarizing solution: superior protection compared with University of Wisconsin solution. J Thorac Cardiovasc Surg. 2000;120:746-54.

3. Jamieson RW, Friend PJ. Organ reperfusion and preservation. Front Biosci. 2008; 13:221-35
4. Yang Q, Zhang RZ, Yim AP, He GW. Histidine-tryptophan-ketoglutarate solution maximally preserves endothelium-derived hyperpolarizing factor-mediated function during heart preservation: comparison with University of Wisconsin solution. J Heart Lung Transplant. 2004;23:352-9.

5. Gross GJ, Peart JN. KATP channels and myocardial preconditioning: an update. Am J Physiol Heart Circ Physiol. 2003;285:H921-30.

6. Testai L, Rapposelli S, Calderone V. Cardiac ATP-sensitive potassium channels: a potential target for an anti-ischaemic pharmacological strategy. Cardiovasc Hematol Agents Med Chem. 2007;5:79-90.

7. Bouchier-Hayes L, Lartigue L, Newmeyer DD. Mitochondria: pharmacological manipulation of cell death. J Clin Invest. 2005;115:2640-7.

8. Saito T, Sato T, Miki T, Seino S, Nakaya H. Role of ATP-sensitive K+channels in electrophysiological alterations during myocardial ischemia: a study using Kir6.2-null mice. Am J Physiol Heart Circ Physiol. 2005;288:H352-7.

9. Fukuzaki K, Sato T, Miki T, Seino S, Nakaya H. Role of sarcolemmal ATP-sensitive $\mathrm{K}+$ channels in the regulation of sinoatrial node automaticity: an evaluation using Kir6.2-deficient mice. J Physiol. 2008;586:2767-78.

10. Yu T, Yu Z, Liu X, Yang S, Ye Y. Myocardial protection with pinacidil induced hyperpolarized arrest during cardiopulmonary bypass. Chin Med J. 2001;114: 1245-8.

11. Diodato MD Jr, Shah NR, Prasad SM, Racen EL, Mizutani S, Lawton JS, et al. Progress towards a more physiologic approach to donor heart preservation: the advantages of hyperpolarized arrest. J Heart Lung Transplant. 2005; 24:1362-8.

12. Yu T, Liu X, Yu Z, Yang S, Ye Y. Myocardial Protection effect of drug preconditioning and hyperpolarized arrest with pinacidil in isolated rabbit hearts. Chin J Surg. 2001;39:401-10.

13. Poderoso JJ, Fernandez S, Carreras MC, Tchercanski D, Acevedo CA, Rubio M, et al. Liver oxygen uptake dependence and mitochondrial function in septic rats. Circ Shock. 1994;44:175-9.

14. Flameng W, Borgers M, Daenen W. Ultrastructural and cytochemical correlates of myocardial protection by cardiac hypothermia in man. J Thorac Cardiovasc Surg. 1980;79:413-24

15. Baczkó I, Giles WR, Light PE. Resting membrane potential regulates $\mathrm{Na}^{+}-\mathrm{Ca}^{2+}$ exchange mediated $\mathrm{Ca}^{2+}$ overload during hypoxia-reoxygenation in rat ventricular myocytes. J Physiol. 2003;550:889-98.

16. Gunter TE, Buntinas L, Sparagna G, Eliseev R, Gunter K. Mitochondrial calcium transport: mechanisms and functions. Cell Calcium. 2000;28:285-96.

17. Yang Q, Zhang RW, Yim YP, He GW. Release of nitric oxide and endotheliumderived hyperpolarizing factor (EDHF) in porcine coronary arteries exposed to hyperkalemia: effect of nicorandil. Ann Thorac Surg. 2005;79:2065-71.

18. Traverse JH, Chen Y, Hou M, Li Y, Bache RJ. Effect of K ${ }^{+}$ATP channel and adenosine receptor blockade during rest and exercise in congestive heart failure. Circ Res. 2007;100:1643-9.

19. Berkich DA, Salama G, LaNoue KF. Mitochondrial membrane potentials in ischemic hearts. Arch Biochem Biophys. 2003;420:279-86.

20. Kowaltowski AJ, Seetharaman S, Pauce P, Garlid KD. Bioenergetic consequences of opening the ATP-sensitive $\mathrm{K}^{+}$channel of heart mitochondria. Am J Physiol Heart Circ Physiol. 2001;280:H649-57.

21. Akao M, Ohler A, O'Rourke B, Marbán E. Mitochondrial ATP-sensitive potassium channels inhibit apoptosis induced by oxidative stress in cardiac cells. Circ Res. 2001;88:1267-75.

22. Moses MA, Addison PD, Neligan PC. Mitochondrial KATP channels in hindlimb remote ischemic preconditioning of skeletal muscle against infarction. Am J Physiol Heart Circ Physiol. 2005;288:H559-67.

23. Crow MT, Mani K, Nam YJ, Kitsis RN. The mitochondrial death pathway and cardiac myocyte apoptosis. Circ Res. 2004;95:957-70.

24. Matsuda N, Morgan KG, Sellke FW. Preconditioning improves cardioplegia-related coronary microvascular smooth muscle hypercontractility: role of KATP channels. J Thorac Cardiovasc Surg. 1999;118:438-45.

25. Rajashree R, Koster JC, Markova KP, Nichols CG, Hofmann PA. Contractility and ischemic response of hearts from transgenic mice with altered sarcolemmal K (ATP) channels. Am J Physiol Heart Circ Physiol. 2002;283:H584-90. 